\title{
Investigation into the micro deep drawing capabilities of a specially engineered refined aluminium alloy
}

\author{
A. Dhal ${ }^{1}$, S.K. Panigrahi ${ }^{1,}{ }^{*}$, and M.S. Shunmugam ${ }^{1}$ \\ ${ }^{1}$ Indian Institute of Technology Madras, Chennai, Tamil Nadu, India - 600036
}

\begin{abstract}
During miniaturisation, size of the part comes close to grain size of the material. There is an overall decrease in the total number grains undergoing deformation and most of these are surface grains. Therefore, microscale deformation is marked by abnormal stress-strain response which limits the manufacturing capabilities of microforming. Two distinct phenomena responsible for this are: (i) dominance of single crystal deformation behaviour, and (ii) increased strain localisation due to incompatibly between surface and core grains during deformation. The present work attempts to neutralise these effects by increasing the number of grains in the deformation zone. This has been achieved by engineering refined microstructure in the materials. To develop the refined microstructure, cryorolling followed by controlled annealing treatment has been employed. Microscale deformation behaviour and microforming capabilities of the refined material have been compared with its coarse-grained counterpart by analysing their tensile curves and by post-mortem study of micro deep drawn components over a wide range of sample thicknesses. Material with fully recrystallised, equiaxed, strain-free refined microstructure is found to have the best strain hardening response both in micro and macro deformation domains. This property is also reflected in the micro deep drawing capabilities of the same material.
\end{abstract}

Keywords: Micro forming, Nano structure, Deep drawing

\section{Introduction}

In the past decade, there has been a visible trend in miniaturisation particularly in the fields of consumer electronics, biomedical devices, avionics etc. The demand of micro components commonly used in such devices is rising in an unprecedented rate. To cater this rising demand, there is a need to escalate the manufacturing process to mass production scale. Currently popular micro manufacturing processes such as photolithography (LIGA) and micro machining are not designed for mass production of complex 3D microparts. On the other hand, microforming can be used for mass production of such intricate products. It also has economic and ecological advantages in terms of assembly-free near net shape production, minimal material wastage, good product accuracy and repeatability, and automation capabilities [1].

Despite its prospects, microforming remains unexploited in micro-components industries. It is because effective material deformation in microscale remains a big challenge. At micro-scale, the deformation behaviour does not follow the conventional trends. This has been attributed to an overall decrease in the total number grains undergoing deformation and an increase in the share of surface grains compared to the core grains [2]. Since surface grains have free unconstrained exteriors, their grain boundaries have limited dislocation accumulation capabilities compared to the core grains. Therefore, incompatibility exists between core and surface grains for dislocation movement, resulting in an increase of strain localisation at their interface. This results in premature necking in the material during microscale deformation and reduced sheet metal forming capabilities [3].
Apart from the challenges associated with the change in material behaviour in micro scale, there are additional hurdles related to the tool development and material handling in case of microforming. The press tool for microforming should have close dimensional tolerances for mating parts, and controlled positioning as well as misalignment errors among them. Unlike macro press tools, these factors are highly critical in nature in case of microtools. Material handling issues such as adhesion of the samples and easy retrieval of the formed component from the die should be considered. These aspects need to be considered while design and development of microforming tools and systems [4].

The total number of grains in the deformation zone can be increased by refining the microstructure to micrometer or sub-micrometer domain. Therefore, use of samples with refined microstructure instead of the conventional coarse-grained microstructure provides a prospective alternative to tackle the size effect related challenges. Significant amount of research has been devoted to study the influence of grain size effect on the microscale deformation behaviour [5-6]. However, to the best of authors' knowledge, there is no evidence of the application of material with refined microstructures in the form of thin sheet for microscale sheet metal operations. In case of sheet metal forming, a plane strain deformation is desirable as deformation along the sheet thickness results in early failure of the component. Therefore, strain localisation in the material is severely detrimental to the forming capabilities of the sheet metal. For successful microscale sheet metal forming, this creates an additional hurdle as microforming is intrinsically susceptible to premature strain localisation. To improve the microformability, thin sheets used for

*Corresponding author: skpanigrahi@iitm.ac.in 
microforming should possess refined microstructure and have good strain hardening ability.

Cryorolling followed by controlled annealing is an efficient process to develop thin sheets with refined microstructure [7]. During cryorolling, dynamic recovery is supressed resulting in preservation of high amount of dislocation density in the material in form of tangles and clusters. Such material has extremely low ductility, poor strain hardening potential and minimal forming capabilities. Controlled annealing of cryorolled sheets progressively results in development of strain free, equiaxed refined grains [8]. At the initial stage of annealing, the randomly intertwined dislocations get detangled, annihilated and rearranged into ordered, lowangled, sub cells. In the subsequent stages of annealing, defect-free, sub-micrometric, high-angled equiaxed grains are recrystallised in the material. Such grains eventually replace the low angled sub cells developed in the initial stages of annealing. However, further annealing results in grain growth and may result in partial and complete loss of the refined microstructure [8].

The present work proposes an experimental methodology to assess the microforming capabilities of cryorolled sheets and understand the relationship between the microstructure and microformability at various stages of annealing. Such investigation is beneficial for optimising the annealing strategies to engineer desirable refined microstructure for improved microformability. In the present work, samples having three distinctively different microstructure have been designed: (i) partially recrystallised refined microstructure (PRR) with large amount of underdeveloped low angled dislocation cells, (ii) completely recrystallised refined microstructure (CRR) with fully developed equiaxed, high angled recrystallised fine grains, (iii) coarse grained microstructure (CG) with fully grown and very coarse grains. To obtain these distinctive microstructures, three different thermomechanical processing routes have been established. First, the micro-scale deformation behaviour of these three different samples have been established by analysing and comparing the tensile stress-strain curves of progressive thinned miniatured samples. Then the micro deep drawing capabilities of the three samples was examined by performing micro deep drawing of the materials, followed by post-mortem analysis of the cross section of the formed micro-cups. These results were correlated with the microstructure of the samples obtained by electron microscopy technique.

\section{Material and methods}

The material used for the present investigation is aluminium AA5083 alloy (Al-93.5\%, Mg-4.5\%, Mn$0.4 \%$, Si- $0.3 \%, \mathrm{Fe}-0.2 \%$ ), which is very popular in sheet metal forming industries because of its good ductility and corrosion resistance. The material has been received in form of $10 \mathrm{~mm}$ thick plates. These plates were first machined into $50 \times 50 \mathrm{~mm}$ square samples and then annealed at a temperature of $450^{\circ} \mathrm{C}$ for 2 hours in an enclosed furnace followed by water quenching to dissolve any solute completely into the matrix and soften the material for cryorolling. The annealed samples were cryorolled by using a 2-high rolling mill $(110 \mathrm{~mm}$ roll diameter) operating at $8 \mathrm{rpm}$. Cryorolling method involved dipping of the base samples in liquid nitrogen bath for 15 minutes followed by its rolling and redipping the rolled specimen in the liquid nitrogen bath. This process was continued for a few numbers of passes until the required reduction has been obtained. The final thickness of the cryorolled specimens was approximately $0.9 \mathrm{~mm}$. These specimens were polished using fine emery paper (grit size $=10 \mu \mathrm{m}$ ) to obtain samples of various thicknesses $(0.1,0.3,0.5$ and $0.7 \mathrm{~mm})$. To obtain samples corresponding to three distinctive microstructures, three different types of annealing treatments were employed. PRR material was obtained after annealing cryorolled samples at $200^{\circ} \mathrm{C}$ for 30 minutes followed by water quenching. CRR material was similarly obtained after annealing cryorolled sheets at $300^{\circ} \mathrm{C}$ for 30 minutes followed by water quenching. Finally, to obtain CG material, the cryorolled samples were annealed at $450^{\circ} \mathrm{C}$ for 12 hours in the furnace followed by water quenching. The selection of these annealing conditions is based on the authors' previous work which investigated the microstructure evolution and grain growth kinetics of the same material at various annealing parameters [8]. The microstructures of the PRR, CRR and CG material were visualised by electron back scattered diffraction (EBSD) technique using a FEI Inspect F50 scanning electron microscope operating at $30 \mathrm{kV}$. To capture the intricate microstructural details of the PRR samples, high resolution micrographs were captured in a Philips CM12 transmission electron microscope (TEM). The samples for the both the electron microscopy processes were prepared using standard techniques (details available in authors' previous work [8]).

Tensile tests were carried for materials with all the three microstructural conditions using a high precision Instron 5984 micro mechanical testing machine equipped with pneumatic grippers. To remove surface roughness and irregularities introduced on the samples in previous stages of polishing, the tensile samples were re-polished using a super-fine emery paper (grit size $=1 \mu \mathrm{m}$ ), followed by electrolytic polishing. A 1:9 acidalcohol solution of $\mathrm{HClO}_{3}+\mathrm{CH}_{3} \mathrm{OH}$ was used for this purpose. To verify the consistency in the sheet thickness, the cross section of the samples was observed under a microscope. Image of one the sample has been shown in fig. 1. Miniature sub-standard specimens (length $=3$ $\mathrm{mm}$, width $=1 \mathrm{~mm}$ ) were used for carrying out the tensile test. The sub-standard dimensions were chosen to capture the micro-deformation behaviour of the material more precisely. The deformation speed of the tensile test was fixed at $0.3 \mathrm{~mm} / \mathrm{min}$. Three tests were performed at each condition for the sake of repeatability.

A custom designed, indigenously developed micro deepdrawing setup was used to evaluate the micro deep drawing capabilities. The tolerances of the press tool components have been controlled well within $5 \mu \mathrm{m}$. An 


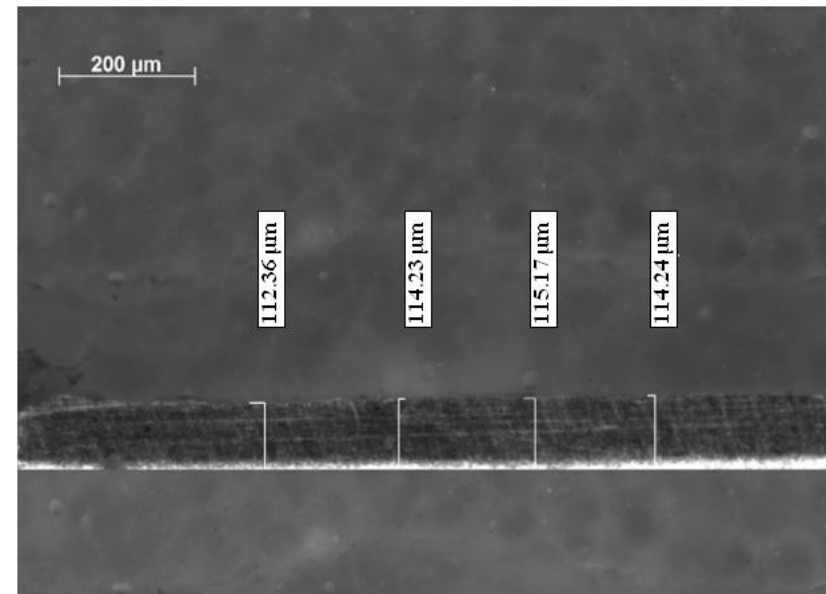

Fig.1. Cross sectional view of the sample with a thickness of $114 \mu \mathrm{m} \pm 2.8 \mu \mathrm{m}$

inverted configuration of micro deep-drawing has been adopted to prevent deflections and improve stability during forming. The diameters of punch and die used in the micro deep drawing tool are $1.5 \mathrm{~mm}$ and $2.0 \mathrm{~mm}$ respectively. A corner radius of $0.5 \mathrm{~mm}$ has been provided in both the punch and die. The photograph of the press tool has been shown in fig. 1. The samples used for micro deep-drawing were first obtained by punching circular blanks (blank diameter $=3 \mathrm{~mm}$, blank thickness 0.1 and $0.3 \mathrm{~mm}$ ) from the PRR, CRR and CG sheets. The samples were then drawn up to a height of $1 \mathrm{~mm}$ in the micro press tool at a forming speed of $0.3 \mathrm{~mm} / \mathrm{min}$. The load-displacement characteristics were recorded using a $2 \mathrm{kN}$ load cell (0.5\% accuracy) and $20 \mathrm{~nm}$ resolution linear encoder. To perform the post mortem analysis of the formed cup, the components (only with thickness of 0.1 and $0.3 \mathrm{~mm}$ ) were embedded in a hardened resin mould, sliced into half to expose their cross section. The polished cross section was visualised under Zeiss STEMI 2000CS stereo microscope and the thickness of the section was measured from the centre to sample edge at an interval of $0.05 \mathrm{~mm}$. The average value was computed by analysing at least 5 samples corresponding to each processing condition. The appearance of the top surface of the micro cups was also observed under the stereo microscope.

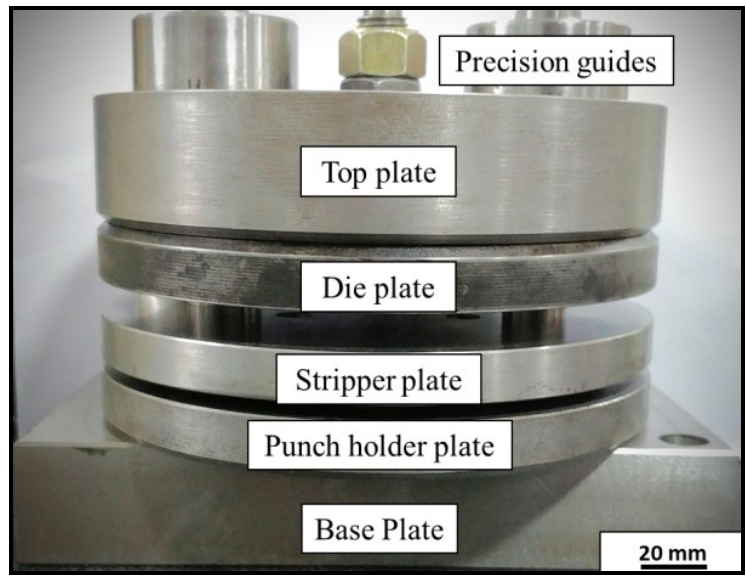

Fig.2. Photograph of the micro deep drawing press tool used in the present investigation

\section{Results and discussions}

\subsection{Microstructural study}

The inverse pole figures of PRR, CRR and CG materials as captured by the EBSD technique has been shown in fig. 3a-3c and the TEM micrograph of the PRR material have been shown in fig. $3 \mathrm{~d}$.

EBSD inverse pole figures reveal significant differences between the PRR, CRR and CG materials in terms of their grain size, morphology and orientation of the grains. Analysis of the EBSD pole figure (fig. 3a) reveals presence of a composite microstructure consisting of both equiaxed and elongated grains. TEM micrograph (fig. 3d) reveals the internal features of the elongated grains as clusters of relatively low angled equiaxed grains (represented as dashed yellow line). Residual dislocations are also found in form of polygonised dislocation walls (red arrows) inside these low angled grains. Dislocation density analysis presented in the authors' earlier paper [8] showed that after annealing at $200^{\circ} \mathrm{C}$, there is an $18.3 \%$ drop in dislocation density of the material compared to the cryorolled state.
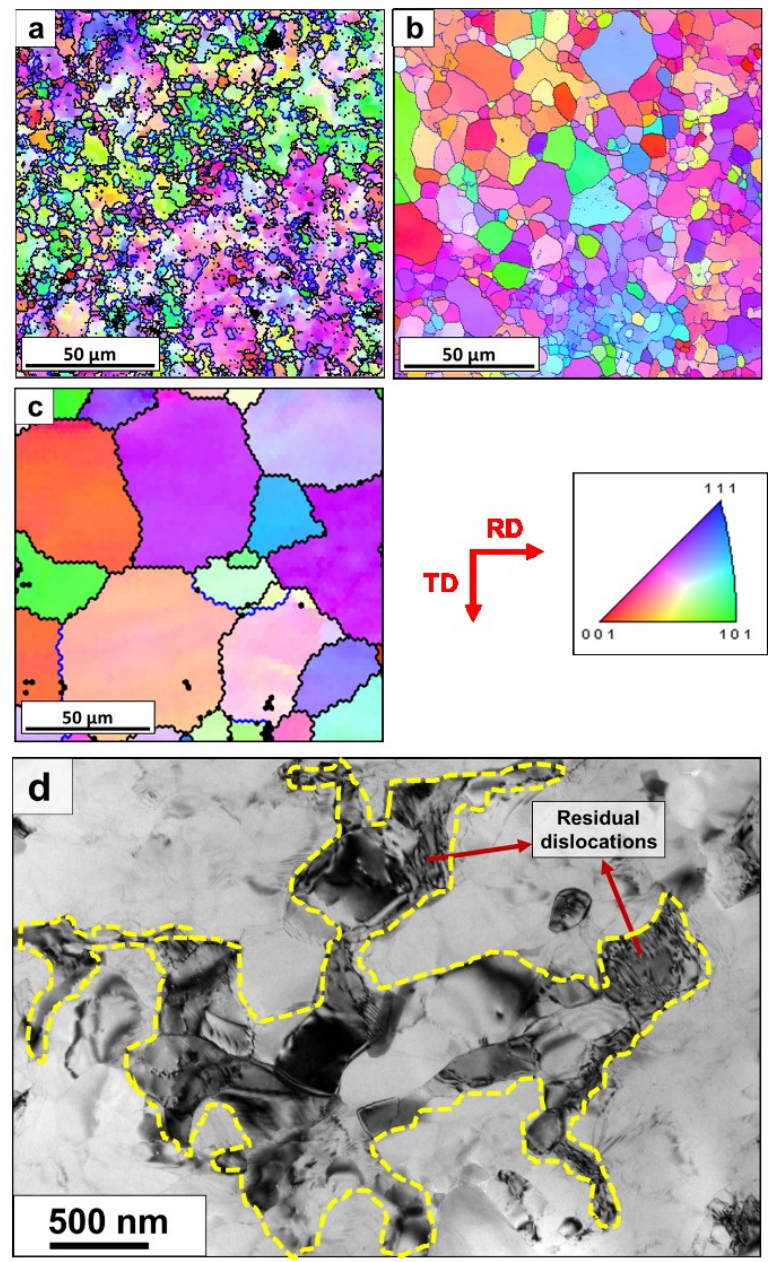

Fig.3. EBSD inverse pole figure of (a) PRR material (b) CRR material (c) CG material (d) High resolution TEM image of the PRR material 
This indicates that annealing at $200^{\circ} \mathrm{C}$ results in annihilation and organisation of dislocation tangles into hierarchical structures comprising of low angled, equiaxed grains embedded inside elongated grains. The average grain size of the selective equiaxed grains is found to be $0.29 \pm 0.12 \mu \mathrm{m}$. EBSD pole figure of the sample annealed at $300^{\circ} \mathrm{C}$ (CRR sample) shows completely recrystallised microstructure, with well defined, equiaxed, high angled grains. Decrease in dislocation density is $86.7 \%$ compared to as-cryorolled condition [8]. Abnormal grain growth results in coexistence of very fine and moderately coarse grains. The average grain size of the refined grains is found to be $3.7 \pm 1.3 \mu \mathrm{m}$. Finally, the EBSD micrograph of the CG material obtained after annealing at $450^{\circ} \mathrm{C}$ for 12 hours reveals fully coarse equiaxed grains. The dislocation density is negligibly low for this material. The average grain size is found to be $88.3 \pm 23.6 \mu \mathrm{m}$.

\subsection{Tensile stress-strain analysis}

Tensile stress-strain curves for all three different materials corresponding to samples of different thicknesses $(0.1,0.3,0.5$ and $0.7 \mathrm{~mm})$ have been compiled in fig 4. To account for the scatter in the stressstrain response, three samples were tested in each condition. The mean values and standard deviations of both yield and ultimate strength have been plotted in fig. 5 .

Two substantial differences can be seen in the stressstrain response of PRR material. First, compared to CG and CRR materials, PRR material has greater yield strength. Second, the ductility and strain hardening potential of PRR material is found to be significantly lower than that of CG and CRR materials. There is a direct correlation between the mechanical properties such as yield strength, ductility and strain hardening capability with their corresponding microstructure. During cryorolling, material has gone through high degree of strain hardening. PRR material obtained after annealing at $200^{\circ} \mathrm{C}$ for 30 minutes, undergoes some level of dislocation annihilation. However, it is still having a fair amount of stored dislocations (in form of low angled dislocation cells and polygonised dislocation boundaries) which obstruction the free motion of dislocation, thereby increasing the material's yield strength. Due to its nanometric grain sizes, the volume fraction of grain boundaries and triple junctions in the PRR material is much greater compared to $\mathrm{CRR}$ and $\mathrm{CG}$ materials. Dislocations travelling from three different directions often get intertwined together and tend to pile up at the triple junctions. Therefore, due to frequent interruptions by triple junctions and residual dislocations in the PRR material, the movement of the newly nucleated dislocations obtained during tensile deformations gets obstructed frequently along the grain boundaries. This results in faster strain localisation and premature fracture of the material, thereby reducing its uniform plasticity/strain hardening potential. As the poor strain hardening is the intrinsic property of the PRR material, this trend is consistently visible throughout all the deformation thicknesses. In microscale, due to very fragile nature of the specimen, the samples of $0.1 \mathrm{~mm}$ thickness fail even faster than the samples of other thicknesses.
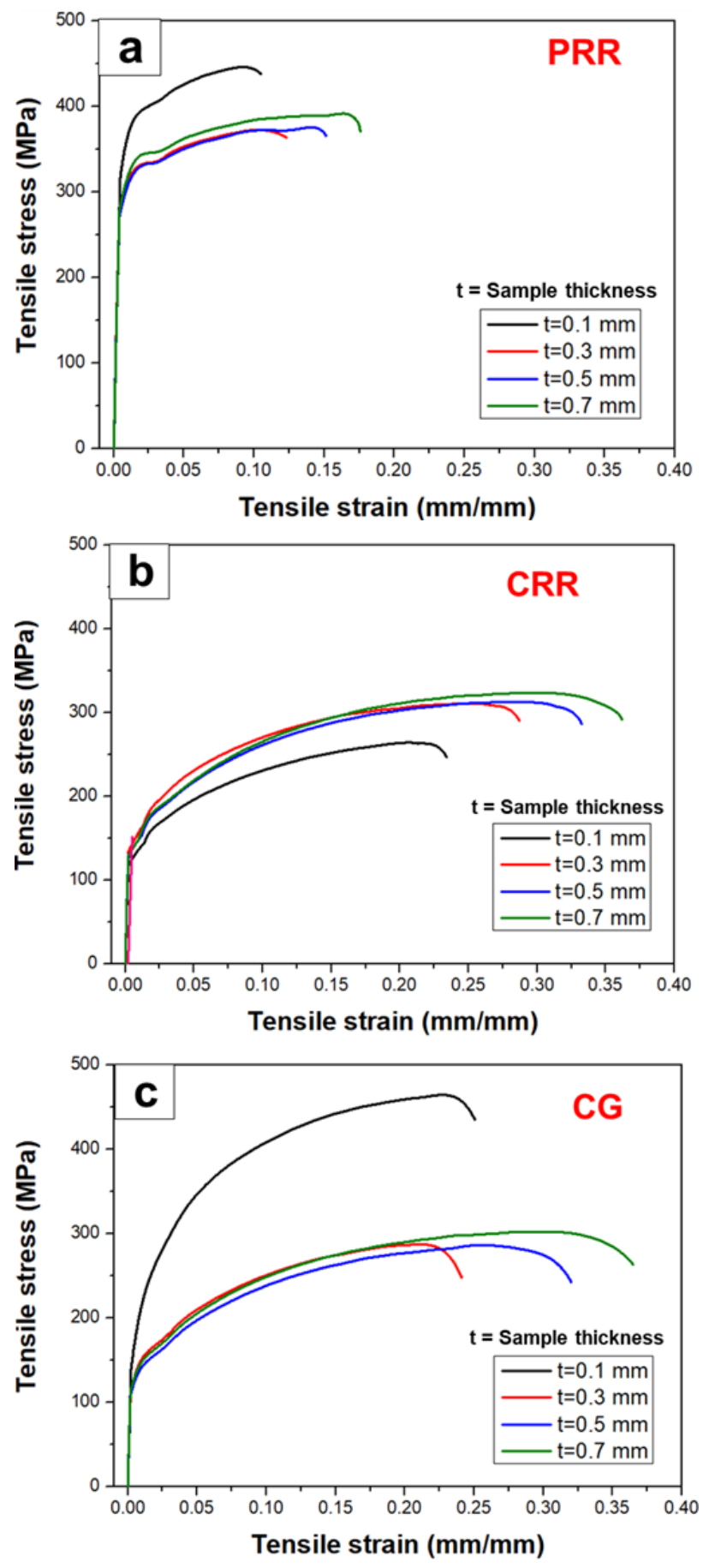

Fig.4. Stress-strain curves for: (a) PRR material (b) CRR material (c) CG material

On the other hand, in case of both CRR and CG material, the ductility and the strain hardening ability of the material is dramatically improved. However, this is achieved by a decrease in both yield and ultimate strength of the materials. The improvement in its strain hardening potential can be associated with the microstructures of the CRR and CG materials. 


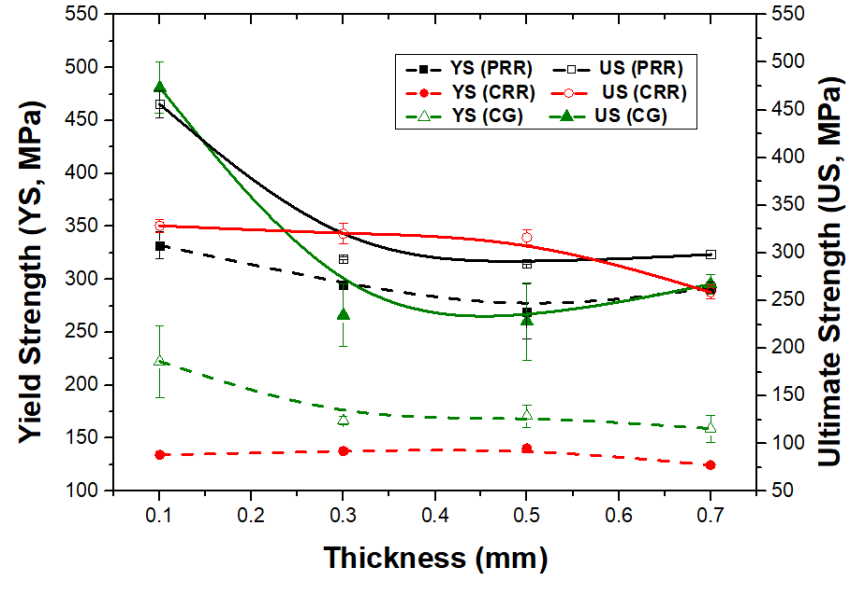

Fig.5. Mean and standard deviations of yield and ultimate strength for various samples

These materials have fully recrystallised and equiaxed grains devoid of excessive residual dislocations. In such microstructures, dislocation motion is relatively easy and free from excessive obstruction by triple junctions.

However, two major deviations in the stress-strain curves are observed between the CG and CRR materials. First, in CG material, with miniaturisation, the ductility is progressively degraded. Second, at microscale (thickness of $0.1 \mathrm{~mm}$ ), there is a sudden upsurge in the stress-strain response. This could be attributed to the size effect during microscale deformation. Since the sample thickness of $100 \mu \mathrm{m}$ is very close to the average grain size of the CG material $88.5 \mu \mathrm{m}$, there is a dominance of single grain deformation mechanism. The large grain is highly resistant towards plastic deformation, which may result in the relatively smaller grains in its vicinity to deform to greater extent. Therefore, the strain distribution is non-uniform and inconsistent leading to poor formability.

\subsection{Evaluation of micro deep drawing capabilities}

Microforming capability is the ability of the material to undergo the required plastic deformation without failure. In micro deep drawing operation, since the thickness of the sheet is much smaller, failure occurs mostly along the sample thickness. In the present work, the crosssectional thickness of the deformed micro components (micro cups) has been measured for samples of PRR, CRR and CG microstructure with an initial sheet thickness of $0.1 \mathrm{~mm}$ and $0.3 \mathrm{~mm}$. The average results compiled from 5 samples corresponding to each processing condition have been shown in fig. 6 .

Since the strain hardening capability of the PRR material is lower compared to CRR and CG materials, it undergoes faster strain localisation. As a result, the material undergoes abrupt thinning in both the cases for PRR sheet. Thinning is prominent along the curvature of the cup. CRR material shows consistently good forming behaviour marked by its good resistance to thinning in both cases. CG material shows good forming behaviour in case of sample thickness of $0.3 \mathrm{~mm}$. However, when
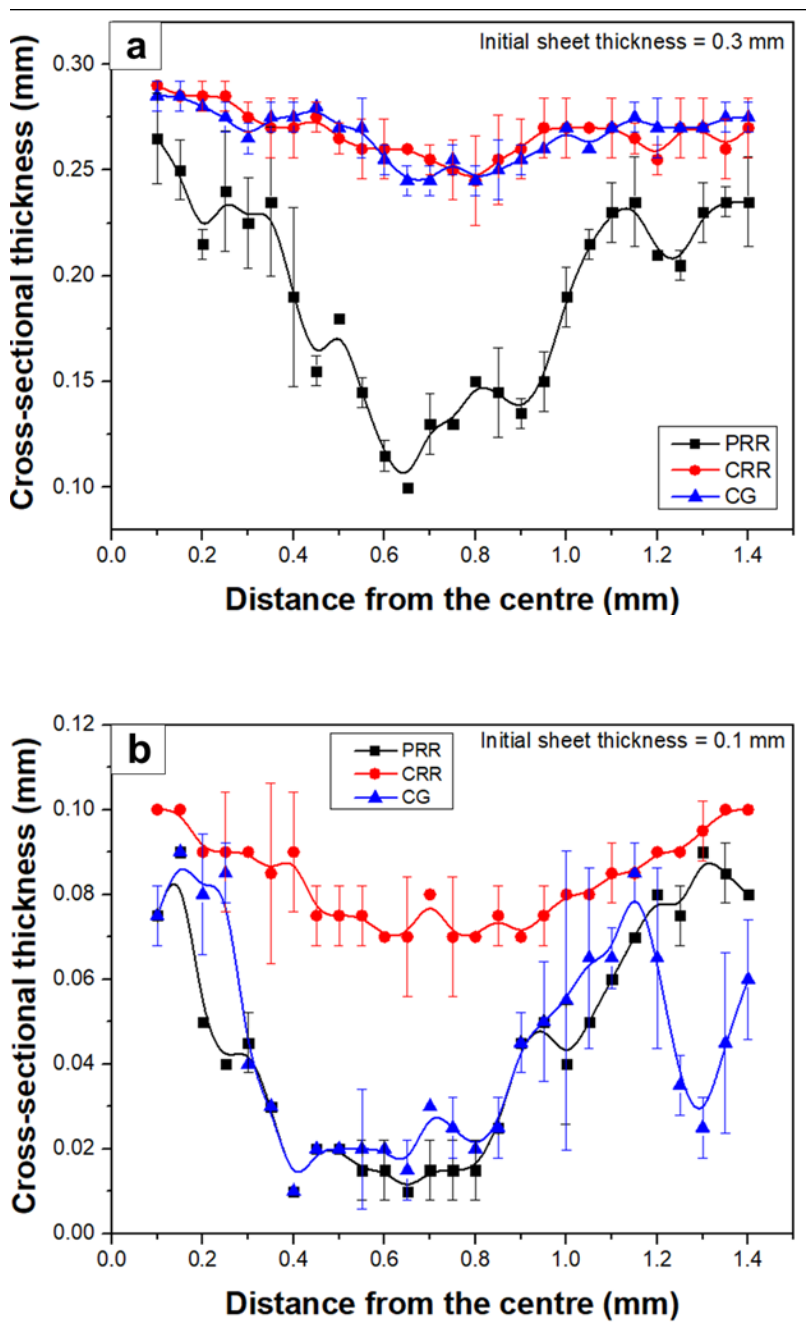

Fig.6. Variation of cross-section thickness of the micro components for an initial sheet thickness of (a) $0.1 \mathrm{~mm}$ (b) $0.3 \mathrm{~mm}$

sample of $0.1 \mathrm{~mm}$ is formed, it shows very erratic distribution of thickness along the deformation zone. This can be attributed to the prominence of single grain deformation which causes incontinent strain distribution and an increase in process scatter. The micro deep drawing capabilities as assessed from the post deformation cross-sectional thickness correlate well with the uniaxial tensile behaviour of the materials.

The appearance of the micro cups has been presented in fig. 7. The micro cups drawn from PRR and CRR materials have visually much smoother surface. On other hand, micro cup drawn from CG material exhibits inferior surface with pronounced undulations. 


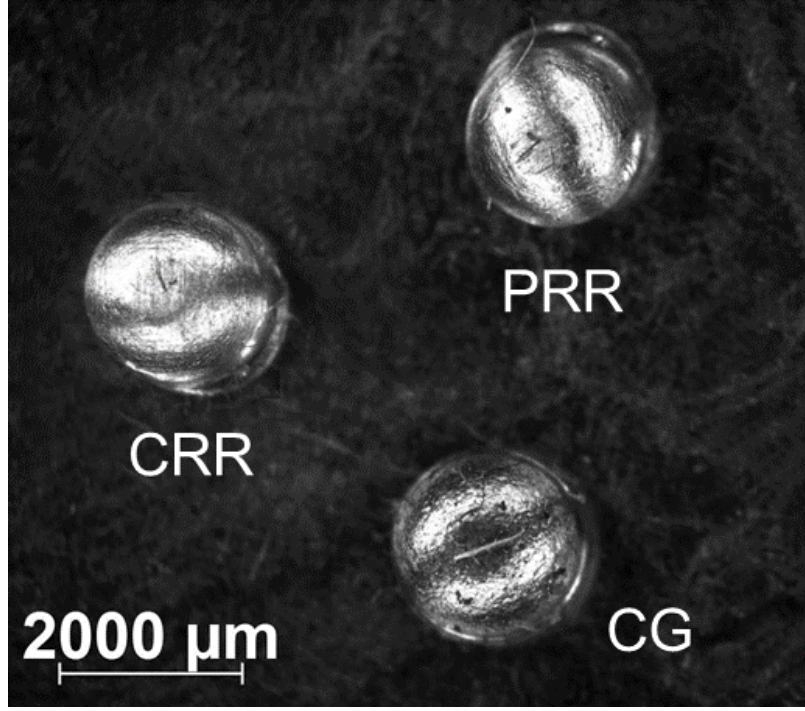

Fig.7. Macrographs of the micro cups drawn from PRR, CRR and CG material

\section{Conclusions}

The microscale deformation behaviour and micro deep drawing capabilities of three different material with varying microstructures (PRR, CRR and CG) have been investigated by tensile testing and post-mortem crosssectional analysis of the deformed micro cups. The PRR material was found to have inferior strain hardening tendency resulting in accelerated strain localisation. This was reflected in its poor micro deep drawing performance. The behaviour has been attributed to two distinctive microstructural features: (i) the intertangling of the dislocation introduced during deformation with the pre-existing residual dislocation in the grain boundaries and (ii) frequent obstruction of the dislocation movement by closely packed triple junctions. The CRR and CG material were found to have significantly greater strain hardening potential. However, when the sample thickness is reduced to $100 \mu \mathrm{m}$, dominance of single grain deformation prevails in the CG material, which results in inconsistent and nonuniform deformation behaviour. CRR material shows consistently good strain hardening behaviour over various deformation scales. This is also reflected in its micro deep drawing behaviour, which shows stable and uniform sheet thickness throughout its cross-section. For CG material, the surface also appears visually inferior as marked by an undulated and rippled surface. On the other hand, for PRR and CRR materials, surfaces appear relatively smoother.

It is clear from the present investigation that refined microstructures have good microforming capabilities at various deformation domains $(0.7 \mathrm{~mm}$ to $0.1 \mathrm{~mm})$. However, in case of microscale sheet metal forming, the microforming capability is highly sensitive to plastic instability. Therefore, refined microstructure with good strain hardening capabilities is desirable. Cryorolling introduces a large amount of dislocation, which requires a certain degree of annealing to relieve. Refined microstructure with dislocation free, equiaxed grains are most suitable for microscale sheet metal forming.

This work was financially supported by Aeronautical Research and Development Board, India (ARDB/01/2031831/M/I). The electron microscopy facility has been funded by FIST grant, Department of Science and Technology, India (SR/FST/ET11059/2012(G)).

We acknowledge the contributions of Mr. D. Ramamoorthy, Applied Engineering \& Technology (P) Ltd, Chennai, in the design and development of micro deep drawing tool used in the present investigation.

\section{References}

1. M. Gieger, M. Kleiner, R. Eckstein, N. Tiesler, U. Engel, CIRP Ann. 50(2), 445-462 (2001)

2. H. Justinger, G. Hirt, J. Mat. Proc. Tech. 209, 21112121 (2009)

3. W.L. Chan, M.W. Fu, J. Lu, Mater. Des. 32(1), 198206 (2011)

4. F. Vollertsen, Z. Hu, H. Schulze Niehoff, C. Theiler, J. Mat. Proc. Tech. 151, 70-79 (2004)

5. M.W. Fu, B. Yang, W.L. Chan, J. Mat. Proc. Tech. 213, 101-110 (2013)

6. J.T. Gau, S. Teegala, K.M. Huang, T.J. Hsiao, B.T. Lin, J. Mat. Proc. Tech. 15, 298-305 (2013)

7. Y. Wang, M. Chen, F. Zhou, E. Ma, Nature 419, 912915 (2002)

8. A. Dhal, S.K. Panigrahi, M.S. Shunmugam, J. Alloy Chem. 726, 1205-1219 (2017) 